\title{
Linear Problems (with Extended Range) \\ Have Linear Optimal Algorithms
}

Edward W. Packel

Department of Computer Science

Columbia University

New York, NY 10027

$$
\text { February } 1984
$$


Linear Problems (with Extended Range) Have Linear Optimal Algorithms

\section{ABSTRACT:}

Let $F_{1}$ and $F_{2}$ be normed linear spaces and $S: F_{0^{--}}>F_{2}$ a linear operator on a balanced subset $F_{0}$ of $F_{2}$. If $\mathrm{N}$ denotes a finite dimensional linear information operator on $F_{0}$, it is known that there need not be a linear optimal algorithm $\phi N\left(F_{0}\right)->F_{2}$ which is optimal in the sense that $\|\phi(N(f))-S(f)\|$ is minimized. We show that the linear problem defined by $\mathrm{S}$ and $\mathrm{N}$ can be regarded as having a linear optimal algorithm if we allow the range of $\phi$ to be extended in a natural way. The result depends upon imbedding $F_{2}$ isometrically in the space of continuous functions on a compact Hausdorff space $X$. This is done by making use of a consequence of the classical Banach-Alaoglu theorem.

\footnotetext{
AMS Subject Classifications:

Primary: 41A45, 41A65, 65J10

Secondary: $46 \mathrm{~B} 99,68 \mathrm{C} 05$
} 


\section{Introduction}

There ha been considerable recent progress in applying and generalizing the information centered theory of optimal algorithms. In particular, when the problem and its information are linear, there are numerous useful and satisfying results Thus, it bas been shown that nonadaptive information is no less powerful (in terms of the error of optimal algonthms) than adaptive information of the same cardinality (see Traub and Wó́niakowski $[4$, p. 49]). Also, for a wide variety of classical linear problems and in several general linear settings, it is known that linear optimal error algorithms exist. While it might seem reasonable to expect, in the light of the above results, that linear problems should always have linear optimal algorithms, there exist specially constructed examples to the contrary

In this paper we resurrect the above intuition that linear problems ought to bave optimal linear algorithms. We do this by showing that, under the minimal requirement that the range of the solution operator is a normed linear space, there must be an optimal linear algorithm if we allow th range to be extended in a natural way. Thus, linear problems do bave linear optimal error algoritbms as long as the solution operator is given an appropriate codomann (perbaps considerably larger than its range).

To develop this result, we will need some machinery from functional analysis, including the classical Banach-Alaoglu theorem. The presentation will be organized as follows. The next section reviews the information centered approach to algorithms in the linear iramework, including some of the existing positive and Degative results The third section introduces additional notation needed to state and discuss the man result. The final section summarizes the technical material needed from functional analysis and proves the mann result

\section{The Information Centered Approach to Linear Problems}

A thorough development of the framework for the information centered approach may be lound in Traub and Wokniakowski [4]. Here we summarize briefly the standard setting for linear problems

Let $F_{1}$ and $F_{2}$ be normed linear spaces over the scalar field $K$, where $K$ is either the real or complex aumbers. Let $F_{0}$ be a balanced gonvex subset of $F_{1}$. In what 
follows, a function with domain $F_{0}$ will be said to be linear if it is the restriction of a lineer lunction defined on $F_{1}$. Given $S: F_{0}->F_{2}$ a linear operator and $N: F_{\sigma^{-}}>K^{*}$ a finite dimensional linear information operator, the inherent error, $r(S, N)$, in approximating the solution $S$ working with the (incomplete) information $N$ is called the radius of information. This important coscept is defined as follows

Given $\left\{\in F_{0}\right.$, let $y=N(\ell)$ and set $V(y)=\left\{g \in F_{0}: N(z)=y\right\}$.

Now define $r(S, N, t)$ to be the radius of $S(V(Y))$ as a subset of $F_{2}$.

Finally, define $r(S, N)=\sup \left\{r(S, N, l):\left\{\in F_{0}\right\}\right.$.

We now investigate a gorithms to aproximate $S(l)$. Since we only bave limited information $y=N(f)$ on $f$, such algorithms can only be defined on $N\left(F_{0}\right)$ of obvious importance are optimal algorithme $: N\left(F_{0}\right)->F_{2}$, where optimality means $\| \alpha(N(t)) \cdot S(I) \mid=r(S, N)$ for all $t \in F_{0}$.

As indicated earlier, we are interested in the existence of linear algorithms which are optimal. There are several reasons why linearity is desirable, which we now summarize. Linear algorithms would appear to be aatural for problems in a linear. setting. Indeed, many of the standard algorithms for classical numerical problems (integration and interpolation, for example) are linear. Linear algorithms tend to be simpler and easier to implement. Most importantly, linear algonthms bave small combinatorial complexity and optimal linear algorithms can be formally shown to have nearly optimal combinatorial complesity (see [4, Chapter 5]). In addition to this valuable efficiency in time, linear algorithms also bave small space complexity (1f we 1gnore precomputation).

Since the result we will develop is immediate when $r(S, N)$ is infinite, we can assume for the remainder of the paper that $r(S, N)<0$. We now state two general positive results concerning the existence of optimal linear algorithms for linear problems. The first theorem covers the case where the solution operator $S$ is scalar-valued.

Theorem 18 If $F_{2}=K$ then there exists a linear optimal error algorithm - $N\left(F_{0}\right)->K$. Thus $\left|0^{\circ}(N(f))-S(f)\right| \leq r(S, N)$ for all $l \in F_{0}$

Proof: The case when $K=R$ is due to Smolyak [2] and can be found in [4. p. 54); the complex case is due to Osipenko [1]

The second result requires a slight reformulation of the general linear problem We can, without los of generality, assume that the balanced, conves set $F_{0}$ on which $S$ and $N$ are defined is senerated by a lineas restriction operator 
$T F_{1}->F_{1}=T\left(F_{1}\right)$ in the sense that $F_{0}=\left\{\left(1 \in F_{1}|T(1)| \leq 1\right\}\right.$

Theocon 82

If $F_{1}=T\left(F_{1}\right)$ is a Hilbert space and $T(\operatorname{tar}(N))$ is closed in $F_{4}$ then there exists a linear optimal error algorithm.

Proon See [4, Chapter 4]. The result emerges in the context of the theory of spline algontbms, and the desired agontbm turns out to be a spline algontbm wacb is central and heace strongly optimal

Theorems 1 and 2 indicate that, in the presence of appropnate structure, optima! linear algontbms cas be constructed. A completely general result along these lines is ruled out by a countererample constructed by Micchelli (see [4], $p$ 60) We sketcb below a somewhat simpler example of a lineas problem which bas no optimal lineas agorithm

Example:

F1x $\lambda \in\left(0, F_{2} / 2\right)$ and let $F_{0}=\left(\left(x_{0}, x_{1}, x_{2}\right): \lambda\left|x_{1}\right|+\left|x_{2}\right| \leq 1,\left|x_{0}\right| \leq\right.$ ?

Let $S: F_{0} \rightarrow R^{2}$ be delined by $S\left(x_{0}, x_{1}, x_{2}\right)=\left(x_{1}, x_{2}\right)$, where

Ris glven lis lilbert nora.

Let $N: R^{2}-\rightarrow Z$ be deflaed by $N\left(x_{0}, x_{1}, x_{2}\right)=x_{1}+\lambda x_{0}$.

De rely bearlly apon Figure 1 , which plctures $S\left(F_{0}\right)$ and $S(V(J))$ for sone critical ralues of $y=N(x)$, where $x=\left(x_{0}, x_{1}, x_{2}\right)$.

Part (a) of the figure 1s siaply for orlentation. It can be cbecked that the broked line bas leagth $<$ lroe which 1 i follons that $S(v(0))$ bas radius 1 .

It can be checked that the radius of the set $S(V(\lambda)$ in part (b) 1s the length of each of the broked lines. Furtbernorg. $S(V(\lambda))$ detarnines $r(S, N)$, Which equals the square root of $1+\lambda$.

O) the vertical $\left(x_{2}\right)$ strnetry of the problea. an lidear optinal a goritbn nust bave the for $(y)=(c y, 0)$ for sone cer.

It then follows fron the above that 19 is to be a linear optinal algoritbr lt nust bave the form $\phi(y)=\left(\lambda^{2}-y, 0\right)$.

Now using part (c) of the l1gure, it can be sbown that witb $y=1 / \lambda-\lambda$. $H(y)-\{1 / \lambda .0), r(S, N)$ ( $C h 1 s$ is were $\lambda \in(0 .(\sqrt{2} / 2)$ is needed). Fe can tbus conclude tbat the stated problea bas no opt inal Ilaear steorien.

\section{(a) S(Kertwresiv(n)}

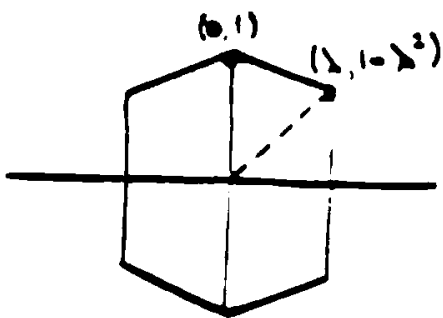

(b) $s(v(\lambda))$

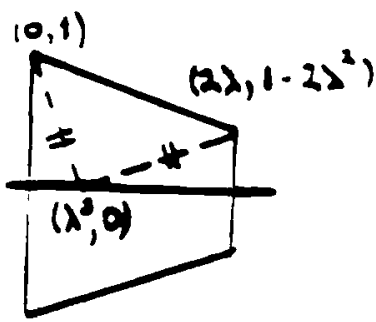

(c) $S(V(\lambda-V \lambda))$

Figure 2-1: The lmage under $S$

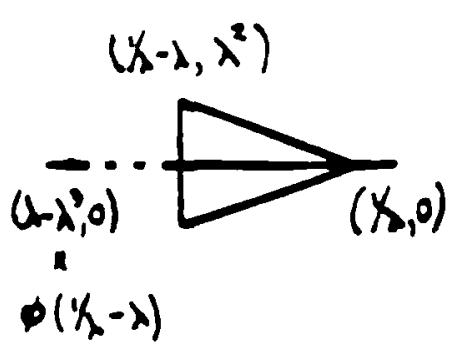


While it is clear that the above example is somewhat contrived, it does show that no general result about optimal linear algoritbms for linear problems in the standard setting is posable. In the next section we show that a small but significant reformulation of the standard linear setting allows for optimal linear algorithms in a very general context.

\section{Optimal Linear Algorithms}

To state our man result, we recall some standard notation from functional analysis. A more complete exposition of these ideas, including proofs of standard results used in this and the next section can be found in Packel [2] (or any other introductory functional analysis text). Given a compact Hausdorff space $X$ define

$C(X)=\{g: X->K / g$ contiauous $\}$

$B(X)=\{g: X->K \mid g$ bounded $\}$

where each function space is endowed with the sup norm

If $E$ and $F$ are normed linear spaces, they are defined to be isometrically isomorphic if there exists a linear bijection $b: E->F$ which is norm-preserving In this case we regard $E$ and $F$ as identical as far as tron normed and linear structures are concerped.

We are now prepared to state the man result

Theorem 8: Given a general linear problem defined by $S$ and $N$, with S. $F_{0} \subseteq F_{1}->F_{2}$, there exists:

i) A compact Hausdorff space $X$ such that $F_{2}$ is isometrically isomorphic to $\hat{F}_{2} \leqq B(X)$

ii) A linear optimal error algorithm $\bullet^{\circ} \mathrm{N}\left(\mathrm{F}_{0}\right)-->B(X)$ satisfying $1 \cdot(N(f))-S(t) \mid \in r(S, N)$ for all $1 \in F_{0}$

Before dereloping the proof, we discuss interpretations of this Theorem. Theorem 1 of the previous section showed that if the range of $S$ is sufficiently simple (namely the scalar field $K$ ), then an optimal linear algorithm was assured. The Example sketched in that section then showed that merely expanding the range of $S$ to $\mathrm{R}^{2}$ destroys the guarantee of an optimal linear algorithm if algorithms are restricted to the range of $S$. Theorem 2 now suggests that by giving $S$ a codomain (namely $B(X)$ ) which extends beyond its range, an optimal linear algorithm (with 
range in thas extended codomana) wust enst.

As the fartbcoming proof will stow, the extended codoman is generally vastly larger and more complicated than the onginal range In addition, its members (otber than the isometnc images from $F_{2}$ ) may have no meaningful connection with the members of $F_{2}$. Nevertheless, one interpretation of the result is that a haear problem does bave an optimal linear algontbon if the solution operator is given an "approprate" codomain. While the theorem uses the ratber extreme case of $B(X)$ for this Eodoman, it may be the case that linear optimality bolds for less drastic extensions of the range of $S$ In particular, it seems reasonable to conjecture. perbaps with added bypotheses, that the Tbeorem might be strengthened by replacing $B(X)$ with $C(X)$. We leave this for now as an open problem.

\section{Technical Background and Prools}

Let $F$ be a normed lineas space over the scalar field $K$ The conjugate apoce $F^{*}$ of $F$ is defined by

$$
F^{*}=\left\{f^{\circ} F->K \mid f^{\circ} \text { continuous and linear on } F\right\}
$$

A natural "operator" norm on $F^{\circ}$ is defined by

$$
\| \mathrm{I}^{\circ} \mathrm{A}=\sup \left\{\left|\mathrm{f}^{\prime \prime}(\mathrm{l})\right|: \| \mathrm{CQ}=1\right\}
$$

A weaker topology on $F^{*}$ can be defined as follows Each fo $F$ induces a linear functional $1^{\circ}$ on $F^{\circ}$ defined by

$$
i^{\circ}\left(f^{*}\right)=f^{*}(f)
$$

The weak ${ }^{*}$ lopology on $F^{*}$ is defined as the weakest topology such that $l^{\circ}$ is continuous for every $!$ in $F$

Under the weak" topology, it thus follows that $1^{\circ} \in \mathrm{F}^{\prime \prime}$ (since $\mathrm{I}^{\wedge}$ is clearly hinear and must be continuous on $F^{*}$ ) Using the natural norms on $F^{*}$ and $F^{\prime \prime}$, we also note that $\left\|I^{\wedge}\right\|=\|I\|$ ladeed. $\left\|I^{\wedge} \mid \leq\right\| f \|$ since

$$
\left\|I^{\wedge}\right\|=\sup \left\{\left|f^{\wedge}\left(f^{*}\right)\right|: \| f^{*} \mid=1\right\}=\sup \left\{\left|f^{*}(f)\right|: \|\left\{^{*} \|=1\right\} \leq \operatorname{oup}\left\{\left\|f^{*}\right\||f|:\left\|f^{*}\right\|=1\right\}=|f|\right.
$$

The lact that $\left\|f^{*}=\right\| f \|$ follows by a routine application of the Habn-Banach theorem on the space $F^{\circ}$ The above result says that $F$ is isometrically isomorphic to a subspace $\hat{F}$ of $F^{\prime *}$ (by meass of the haear isometry ":F- $P F^{2 *}$ ) We no: apply these ideas to state and prove the following "folk" result about normed linear spaces Though this result can be found in a varlety of texts, we give the proof here since it 1 s short and sets the stage for the ain theorem. 
Lemma 4: Let $F$ be a normed linear space. Then there exists a compact Hausdorff space $X$ such that $F$ is isometrically isomorphic to a subspace $\widehat{F}$ of $C(X)$. If $F$ is a Banach space, then $\widehat{F}$ is a closed subspace of $\mathbf{C}(\mathbf{X})$.

Proof: Give the conjugate space $F^{*}$ its weak" topology Let $X$ be the unit ball of $F^{*}-X=\left\{f^{*} \in F^{*}: f^{*} \| \leq 1\right\}$. The classical Banach-Alaoglu theorem says that $X$ is compact in the weak topology on $X$. Define $\cdot F->C(X)$ by $f \mapsto l^{\circ}$, where $l^{\prime}\left(f^{\circ}\right)=f^{\prime}(f)$ for all $f^{\prime} \in X$. Then, as developed above, $\left\|f^{*} I=V I\right\|$, so the subspace $\widehat{F}$ of $C(X)$ defined by $\widehat{F}=\left\{f^{\circ} t \in F\right\}$ is isometrically isomorphic to $F$. If $F$ is a Banach space, then $F$ and $\widehat{F}$ are complete, making $\widehat{F}$ closed as a complete subspace of $C(X)$.

Remark The above result is not as powerful as it may seem at first glance, since very little is known about the subspaces of $C(X)$. Our application to the prool of Theorem 2 is, to our knowledge, the first really meaningful use of this curious result. Before proceeding, we note that we can also treat $F$ as being isometrically imbedded in $B(X)$ since $C(X) \leq B(X)$.

We now prove the main theorem which we restate for the convenience of the reader

Theorem Given a general linear problem define: by $S$ and $N$, with $S F_{0} S F_{1}->F_{2}$, there exists

1) A compact Hausdorff space $X$ such that $F_{2}$ is isometrically isomorphic to $\widehat{F}_{2} \leqslant B(X)$

11) A linear optimal error algorithm $: N\left(F_{0}\right)-->B(X)$ satisfying $\|\cdot(N(f))-S(f)\| \in(S, N)$ for all $f \in F_{0}$.

Proof: 1) The existence of $\mathrm{X}$ and $\widehat{F}_{2}$ follow directly from the Lemma proved previousiy.

ii) For each fixed $x \in X$, consider the linear problem

$$
S_{x} \cdot F_{0}>K \text { where } S_{x}(f)=\widehat{S(f)}(x)
$$

By Theorem 1 we know there exists a linear optimal algorithm ${ }_{x} N\left(F_{0}\right)->K$ such that

$$
\left\|\phi_{x}(N(f))-S_{x}(f)\right\| \leqslant r\left(S_{x}, N\right) \leq r(S, N) \text { for all } f \in F_{0}
$$

Letting $x$ vary over $X$ we now must show that the linear operator $\dot{\circ}^{\circ}$ thus defined on $N\left(F_{0}\right)$ bas its range in $B(X)$. First observe that 


$$
\left|S_{x}(f)\right|=|\widehat{S(f)}(x)| \leq\|\widehat{S(t)}\|=\eta S(f) \|
$$

where the inequality follows from $\|\widehat{S(l)}\|=\sup \{|\widehat{S(f)}(x)| x \bullet X\}$. Using (1) and (2), we bare for all $\in \in F_{0}$

$$
\begin{aligned}
& \left|\bullet^{\circ}(N(f))(x)\right| \leqslant\left|0^{\prime \prime}(N(f))(x)-S_{x}(f)\right|+\left|S_{x}(f)\right| \\
& \leq\left|0_{x}^{*}(N(f))-S_{x}(f)\right|+\left|S_{x}(f)\right| \\
& \leq r(S, N)+\| S(I) \mathbb{R}
\end{aligned}
$$

Since the linal expression is independent of $x,(N(f)) \in B(X)$ and the proof is complete.

Acknowledgement: The author wishes to thank Henryk Wo'źnakowski lor his belpful suggestions on organizing these results. 


\section{References}

[1] K. Yu. Osipenko, Best approrimation of analytic functions from information about their ralues at a finite number of points, (Englisb translation) Matb. Notes, $10(1976), 17.23$

(2) E. W. Packel, Functional Analysis: a Short Course, Kreger, 1980

(2) S. A. Smolyak, On optimal restoration of functions and functionals of them (in Russian), Candidate Dissertation, Moscow State Univ. (1965).

[4] J. F Traub and H. Wó́niakowski, A General Theory of Optimal Algoritbms, Academic Press, 1980 